\title{
Methods of assessing associated reactions of the upper limb in people with stroke and traumatic brain injury: A systematic review
}

Michelle B.Kahn, Benjamin F.Mentiplay, Ross A. Clark, Kelly J. Bower \& Gavin Williams

\section{SUPPLEMENTARY MATERIAL}

\section{Appendix 1. Taxonomy for Associated Reactions}

A thorough and systematic literature search in the field of associated reactions (ARs) has highlighted the problems with taxonomy and nomenclature in describing this phenomenon. The lack of a standardized definition of the term 'associated reactions', problems with assessment and controversy over contributing factors are issues that are long-standing and are largely unchanged over the last two decades. ${ }^{1-3}$ A number of terms exist in the literature that overlap with or are utilised interchangeably when referring to ARs. The terms most frequently used are 'motor overflow', 'associated movements', 'global sykinesis' and 'mirror movements'. Clarification of these terms is essential for the purposes of this review, for clinical application and for effective communication within and between professional groups involved in the rehabilitation of people with brain injury.

Associated reactions are pathological and occur due to damaged supraspinal structures as a result of neurological injury. Associated reactions are involuntary postural reactions which are triggered by effort. ${ }^{4}$ The involuntary movement occurs globally in heterogeneous muscles on the hemiplegic side, coinciding with, or following effort exerted at another body site. ${ }^{5,6}$ Associated reactions interfere with the recovery of function and the ability to perform efficient and effective movement. ${ }^{2}$ Another term, 'global synkinesis', appears to be synonymous with ARs. ${ }^{7,8}$ Global synkineses have been shown to occur in people with acquired brain injury in fairly consistent stereotyped patterns and are most readily elicited in the affected upper limb of people with hemiparesis during voluntary, forceful, resisted contractions on the contralateral side. ${ }^{7,8}$

There are other terms which are related to, but distinct from ARs. 'Motor overflow' is an umbrella term used to define all unintended muscle activity that may be present as overt or covert movement that co-occurs with voluntary movement. ${ }^{9-11}$ Not all of these unwanted upper limb movements are pathological ARs. 'Associated movements' are a form of motor overflow. Associated movements are a normal phenomenon during childhood development until the motor overflow is brought under inhibitory control of the central nervous system by the end of the first decade of life. ${ }^{12}$ They are thought to result from overflow of neuronal excitation across the cortex or spinal cord during volitional movement. ${ }^{13}$ Associated movements may also be a common occurrence observed in intact healthy adults during completion of a complex task or exertion of maximum force or speed. ${ }^{14,15}$ For example, a person's tongue may protrude whilst performing a complex action or learning a new skill. By comparison, 'associated reactions' are always pathological whilst 'associated movements' are normal motor activity that occurs in response to stress. ${ }^{15}$

'Mirror movements' or 'imitative synkineses' are a specific type of motor overflow. They are also a normal component of childhood development but may recur in pathological clinical conditions such as cerebral palsy, Parkinson's disease, Huntington's disease and stroke. ${ }^{16-18}$ An example of a mirror movement is if a person grasps an object in one hand and the other hand may flex into a grip position as well. ${ }^{19}$ Mirror movements are mirror reversals of the intended movement confined to an unintentional movement in the same muscle on the opposite side. They occur predominantly at distal segments in homonymous muscles, not necessarily on the affected side. ${ }^{20,}{ }^{19}$ In contrast, ARs are always a pathological form of motor overflow and are confined to the hemiplegic upper limb. 
For the purpose of this review, the focus will be on ARs in people with cerebral damage as a result of stroke or traumatic brain injury. Given the problems with taxonomy and nomenclature, the working definition for this systematic review will be; 'Associated reactions are an unwanted, effort dependent limb movement that occurs following cerebral damage, where there may be sensorimotor dysfunction or insufficient postural control, so that when a stimulus is applied that goes beyond the individual's level of inhibitory or modulatory control, it results in intermittent or sustained involuntary, heterogeneous muscle activation with abnormal limb posturing, most visible in the hemiplegic upper limb.'

\section{References:}

1. Stephenson, R., S. Edwards, and J. Freeman, Associated reactions: their value in clinical practice? Physiotherapy Research International, 1998. 3(1): p. 69-75.

2. Ellerington, M.E., Letters to the Editor. Physiotherapy Research International, 1998. 3(1): p. 76-78.

3. Shepherd, R., Stephenson et al. - a response. Physiotherapy Research International, 1998. 3(1): p. 78-81.

4. Walshe, F.M.R., On certain tonic or postural reflexes in hemiplegia, with special reference to the so-called "associated movements.". Brain, 1923. 46(1): p. 1-37.

5. Dickstein, R., Y. Heffes, and N. Abulaffio, Electromyographic and positional changes in the elbows of spastic hemiparetic patients during walking. Electroencephalography and clinical neurophysiology, 1996. 101(6): p. 491-496.

6. Dvir, Z. and E. Panturin, Measurement of spasticity and associated reactions in stroke patients before and after physiotherapeutic intervention. Clinical Rehabilitation, 1993. 7(1): p. $15-21$.

7. Boissy, P., et al., Characterization of global synkineses during hand grip in hemiparetic patients. Archives of Physical Medicine and Rehabilitation, 1997. 78(10): p. 1117-1124.

8. Boissy, P., et al., Effects of upper and lower limb static exertions on global synkineses in hemiparetic subjects. Clinical Rehabilitation, 2000. 14(4): p. 393-401.

9. Addamo, P.K., et al., The effects of age and attention on motor overflow production-A review. Brain Research Reviews, 2007. 54(1): p. 189-204.

10. Armatas, C.A., J.J. Summers, and J.L. Bradshaw, Mirror movements in normal adult subjects. Journal of clinical and experimental neuropsychology, 1994. 16(3): p. 405-413.

11. Hoy, K.E., et al., Investigating the cortical origins of motor overflow. Brain Research Reviews, 2004. 46(3): p. 315-327.

12. Lazarus, J.A.C. and J.I. Todor, Age differences in the magnitude of associated movement. Developmental Medicine \& Child Neurology, 1987. 29(6): p. 726-733.

13. Ada, L. and N. O'Dwyer, Do associated reactions in the upper limb after stroke contribute to contracture formation? Clinical Rehabilitation, 2001. 15(2): p. 186-194.

14. Hwang, I.-S., et al., Electromyographic analysis of joint-dependent global synkinesis in the upper limb of healthy adults: Laterality of intensity and symmetry of spatial representation. Journal of Electromyography and Kinesiology, 2006. 16(4): p. 313-323.

15. Bobath, B., Adult hemiplegia: evaluation and treatment. 1990: Butterworth-Heinemann London.

16. Koerte, I., et al., Mirror movements in healthy humans across the lifespan: effects of development and ageing. Developmental Medicine \& Child Neurology, 2010. 52(12): p. 1106-1112.

17. Addamo, P.K., et al., Relative or absolute? Implications and consequences of the measures adopted to investigate motor overflow. Journal of Motor Behavior, 2011. 43(3): p. 203-12.

18. Cox, B.C., M. Cincotta, and A.J. Espay, Mirror movements in movement disorders: a review. Tremor and Other Hyperkinetic Movements, 2012. 2.

19. Uttner, I., et al., Quantitative evaluation of mirror movements in adults with focal brain lesions. Eur J Neurol, 2005. 12(12): p. 964-75.

20. Wittenberg, G.F., et al., Mirror movements complicate interpretation of cerebral activation changes during recovery from subcortical infarction. Neurorehabilitation \& Neural Repair, 2000. 14(3): p. 213-21. 


\section{CINAHL}

\#1 MESH: (MH "Stroke") OR (MH "Stroke, Lacunar") OR (MH "Subarachnoid Hemorrhage") OR (MH "Hematoma, Subdural") OR (MH "Cerebral Hemorrhage") OR (MH "Intracranial Hemorrhage") OR (MH "Basal Ganglia Hemorrhage") OR (MH "Sinus Thrombosis, Intracranial") OR (MH "Intracranial Thrombosis") OR (MH "Intracranial Embolism") OR (MH "Intracranial Embolism and Thrombosis") OR (MH "Intracranial Arterial Diseases") OR (MH "Central Nervous System Diseases") OR (MH "Cerebral Arterial Diseases") OR (MH "Carotid Artery Diseases") OR (MH "Basal Ganglia Cerebrovascular Disease") OR (MH "Cerebral Ischemia")

\#2 ABSTRACT: stroke OR “cerebrovascular accident" OR CVA OR "cerebral infarct*” OR "brain infarct*" OR "intracranial haemorr*" OR "intra-cranial haemorr*" OR "intracranial hemorr*" OR "intra-cranial hemorr*" OR "cerebral haemorr*" OR "cortical haemorr*" OR "cortical ischaem*" OR "cerebral ischaem*" OR "cerebral ischem*" OR "brain ischaem*" OR "brain ischem*" OR "cerebrovasc* disord*" OR "cerebral aneurysm*" OR "brain aneurysm*" OR "hypoxic brain injur*” OR "anoxic brain injur*" OR "subdural haem*” OR "subdural Hem*" OR "intracranial thromb*" OR "intra-cranial thomb*" OR "intra-cranial emb*" OR "intracranial emb*" OR "brain lesion*" OR "cerebral lesion*" OR "cortical lesion*"

\#3: TITLE: stroke OR "cerebrovascular accident" OR CVA OR "cerebral infarct*" OR "brain infarct*" OR "intracranial haemorr*" OR "intra-cranial haemorr*" OR "intracranial hemorr*" OR "intra-cranial hemorr*" OR "cerebral haemorr*" OR "cortical haemorr*" OR "cortical ischaem*" OR "cerebral ischaem*" OR "cerebral ischem*" OR "brain ischaem*" OR "brain ischem*" OR "cerebrovasc* disord*" OR "cerebral aneurysm*" OR "brain aneurysm*" OR "hypoxic brain injur*" OR "anoxic brain injur*" OR "subdural haem*" OR "subdural Hem*" OR "intracranial thromb*" OR "intra-cranial thomb*" OR "intra-cranial emb*" OR "intracranial emb*" OR "brain lesion*” OR "cerebral lesion*" OR "cortical lesion*"

\#4 MESH: (MH "Brain Injuries") OR (MH "Right Hemisphere Injuries") OR (MH "Left Hemisphere Injuries") OR (MH "Hypoxia-Ischemia, Brain") OR (MH "Hypoxia-Ischemia, Brain, Neonatal") OR (MH "Brain Concussion") OR (MH "Head Injuries") OR (MH "Hypoxia, Brain")

\#5 ABSTRACT: "traumatic brain injur*" OR TBI OR "acquired brain injur*” OR ABI OR "Diffuse Axonal Injur*" OR "cerebrovascular trauma" OR "brain damage" OR "brain injur*" OR "cerebral trauma" OR "cerebral damage" OR "head injur*"

\#6 TITLE: "traumatic brain injur*" OR TBI OR "acquired brain injur*" OR ABI OR "Diffuse Axonal Injur*" OR "cerebrovascular trauma" OR "brain damage" OR "brain injur*" OR "cerebral trauma" OR "cerebral damage" OR "head injur*"

MESH: (MH "Nervous System Diseases") OR (MH "Diagnosis, Neurologic")

ABSTRACT: "neurolog*" OR "neuro* dis*” OR "central nervous system dis*" OR "CNS dis*" OR "nervous system dis*"

TITLE: "neurolog*" OR "neuro* dis*" OR "central nervous system dis*" OR "CNS dis*" OR "nervous system dis*"

\#10 MESH: (MH "Hemiplegia") OR (MH “paralysis")

\#11 ABSTRACT: hemipleg* OR hemipar* 
\#12 TITLE:_hemipleg* OR hemipar*

\#13 ABSTRACT: "associated reaction*" OR "associated movement*" OR "global synkines*" OR "Motor overflow" OR "Mirror movement*"

\#14 TITLE: "associated reaction*” OR "associated movement*" OR "global synkines*” OR "Motor overflow" OR "Mirror movement*"

\#15 \#1 OR \#2 OR \#3 OR \#4 OR \#5 OR \#6 OR \#7 OR \#8 OR \#9 OR \#10 OR \#11 OR \#12

\#16 \#13 OR \#14

\#17 COMBINED SEARCHES \#15 AND \#16 
\#1 POPULATION: stroke OR (cerebrovascular accident) OR CVA OR (cerebral infarct*) OR (brain infarct*) OR (intracranial haemorr*) OR (intra-cranial haemorr*) OR (intracranial hemorr*) OR (intra-cranial hemorr*) OR (cerebral haemorr*) OR (cortical haemorr*) OR (cortical ischaem*) OR (cerebral ischaem*) OR (cerebral ischem*) OR (brain ischaem*) OR (brain ischem*) OR (cerebrovasc* disord*) OR (cerebral aneurysm*) OR (brain aneurysm*) OR (hypoxic brain injur*) OR (anoxic brain injur*) OR (subdural haem*) OR (subdural Hem*) OR (intracranial thromb*) OR (intra-cranial thomb*) OR (intra-cranial emb*) OR (intracranial emb*) OR (brain lesion*) OR (cerebral lesion*) OR (cortical Lesion*) OR (traumatic brain injur*) OR TBI OR (acquired brain injur*) OR ABI OR (Diffuse Axonal Injur*) OR (cerebrovascular trauma) OR (brain damage) OR (brain injur*) OR (cerebral trauma) OR (cerebral damage) OR (head injur*) OR (neurolog*) OR (neuro* dis*) OR (central nervous system dis*) OR (CNS dis*) OR (nervous system dis*) OR Hemipleg* OR hemipar*

ASSOCIATED REACTION TERMS: "associated reaction*" OR "associated movement*" OR "global synkines*" OR "Motor overflow"

PSYCHOMETRIC TERMS: Reliability OR validity OR psychometric* OR clinometric* OR "outcome measure" OR "outcome assessment"

\#4 METHOD OF ASSESSMENT (1): (subjective rating scale*) OR (clinician rating scale*) OR (clinician rat* form) OR (clinician rat*) OR (associated reaction rating scale) OR (patient rating scale*) OR (patient rat*)

METHOD OF ASSESSMENT (2): electromyography OR EMG OR (surface EMG) OR (surface electromyography) OR SEMG

METHOD OF ASSESSMENT (3): (strain gauge dynamo*) OR (biodex dynamo*) OR (isokinetic dynamo*) OR (hand held dynamo*) OR HHD

METHOD OF ASSESSMENT (4): goniomet* OR electrogoniomet*

\#10 \#1 AND \#2 AND \#3 AND \#6

\#10 \#1 AND \#2 AND \#3 AND \#7 